\title{
The Measurement of Intellectual Influence: the Views of a Sceptic*
}

\author{
Roberto Serrano ${ }^{\dagger}$ \\ Working Paper No. 2004-02 \\ January 2004
}

\begin{abstract}
In an extremely interesting paper, Palacios-Huerta and Volij (2004) [PV] introduce the axiomatic method to the problem of how to rank academic journals on the basis of their mutual citations. They characterize the invariant method as the only one satisfying a list of five appealing properties. In this note, I show an impossibility result, by identifying a sixth property that is violated by the invariant method. Further, I question the appeal of the PV axioms, when applied over larger domains of problems that take into account making distinctions among types of citations.
\end{abstract}

JEL classification numbers: A0.

Keywords: journal rankings, invariant method, impossibility result, axiomatic method.

\footnotetext{
${ }^{*}$ I am indebted to Pierre Menard, Ignacio Palacios-Huerta and Oscar Volij for introducing me to the topic and for helpful comments. I also remember at this time those colleagues who make out of the art of counting one of their main daily occupations. Research support from National Science Foundation under grant SES-0133113 and Deutsche Bank is acknowledged.

†Brown University, roberto_serrano@brown.edu, www.econ.brown.edu/faculty/serrano
} 


\section{Introduction}

In an extremely interesting paper, Palacios-Huerta and Volij (2004), PV in the sequel, introduce the axiomatic method to the problem of ranking journals as a function of their citations. They rightly criticize previous attempts to reduce all the information contained in scholarly publications to a simple ranking. Indeed, they point out that all rankings available prior to theirs violate at least one of the axioms they propose.

Their main result is the characterization of the invariant method as the only one that satisfies a list of five reasonable axioms:

anonymity, i.e., the ranking should not depend on the names of the journals;

invariance to citation intensity, i.e., all other things equal, the ranking should be unaffected by the length of the reference section in the papers published by a journal;

weak homogeneity, i.e., the relative ranking of any two journals should be a function of their mutual citations;

weak consistency, i.e., the ranking method should be "consistent" when applied to problems involving different numbers of journals; and

invariance to splitting of journals, i.e., if a journal is subdivided into two identical subjournals in terms of their citations, each of the two receives half the original weight of the mother journal, while the valuations of all other journals are unaffected.

All these are reasonable principles in the domain of problems studied by PV. However, when introducing axiomatics, some problems call for an impossibility result, and I believe this is one such problem. Indeed, I propose a sixth reasonable property that is violated by the invariant method, thereby obtaining an impossibility result. We also question the appeal of the PV axioms when used over larger domains that take into account different kinds of citations.

In general, I agree with PV that neither the invariant method nor any other can substitute for the expert judgement and sound opinion of scholars. My analysis is therefore a complement to that of PV.

\section{The Model}

Let $\mathcal{J}$ be a non-empty set of journals. Let $J \subset \mathcal{J}$ be a subset of journals. A citation matrix for $J$ is a $(|J|,|J|)$ non-negative matrix $C=\left(c_{i j}\right)$, where for each $i, j \in J, c_{i j}$ is the citations to journal $i$ by journal $j$ (i.e., the number of times that articles published in journal $i$ are cited by articles published in journal $j$ ). It is assumed that $C$ is irreducible. Define $D$ to be the diagonal matrix, whose $(j j)$-th term is $\sum_{i \in J} c_{i j}$. Define $A$ to be the diagonal matrix, whose $(j j)$-th term is $a_{j}$, the number of articles published by journal $j$. 
We shall refer to the triple $R \equiv\langle J, A, C\rangle$ as a ranking problem, where $J \subset \mathcal{J}$. Let $\mathcal{R}$ be the set of all ranking problems.

A ranking method $\phi$ assigns to each ranking problem $R=\langle J, A, C\rangle \in \mathcal{R}$ a vector $\phi(R) \in \Delta_{J}$, where $\Delta_{J}$ is the unit simplex over $J$. Thus, the vector $\phi(R)$ provides relative valuations of the journals in $J$ as a function of the information contained in $A$ and $C$.

The invariant method is the following ranking method: $\phi_{I}(R)$ assigns to each ranking problem $R$ the vector $v \in \Delta_{J}$ satisfying

$$
\left(I-A^{-1} C D^{-1} A\right) v=0 .
$$

PV argue forcefully in favor of the invariant method. In particular, they demonstrate that it is the only ranking method that satisfies anonymity, invariance to citation intensity, weak homogeneity, weak consistency and invariance to splitting of journals. All these properties can be justified in the domain of problems under study, and PV successfully do so.

\section{An Impossibility Result}

Our first observation is that one could think of an additional appealing property that the invariant method violates, thereby yielding an impossibility result.

Example 1 Consider the following ranking problem $R=\langle J, A, C\rangle$, where $J=\{1,2\}$, $A$ is such that $a_{j}=a$ for $j=1,2$, and $C$ is the matrix with the constant $k$ in every entry. Clearly, the invariant method assigns the vector $(1 / 2,1 / 2)$ to this problem, which is reasonable. Consider now the addition of a third journal, which also publishes $a$ articles, and suppose that the new citation matrix $C^{\prime}$ has the following entries:

$$
\left(\begin{array}{lll}
k-1 & k & 1 \\
k & k & 0 \\
1 & 0 & 2 k-1
\end{array}\right) .
$$

To make our point clear, the reader should think of $k$ as being a large constant. One can calculate that the invariant method assigns to the expanded problem the vector $(1 / 3,1 / 3,1 / 3)$, which does not appear reasonable. The same conclusion is reached if the original set of journals consists of an arbitrary number, $|J|$, of journals, instead of only two.

The example illustrates the poor behavior of the invariant method "near a reducible problem." The reason is that the method, as defined, need not be continuous at reducible points: for reducible matrices, there are multiple solutions to the stationary equation that defines the invariant method, and therefore, the mapping of solutions to the equation is 
not a lower hemicontinuous correspondence at those points. ${ }^{1}$ We shall call journals like the new journal in Example 1 "boundary journals."

Furthermore, the example can be modified to get rid of the zeros, because of the continuity of the invariant method on the domain of irreducible problems. This means that there will exist an open ball of irreducible matrices around that of Example 1 in which the invariant method weights will be far from measuring true influence.

consider the following axiom:

Limited influence of Boundary Journals: ${ }^{2}$ Let $R \equiv\langle J, A, C\rangle$ be a ranking problem where $A=a I_{J}$ and $C$ is such that $c_{i j} / \sum_{i \in J} c_{i j}$ is in an $\epsilon$-neighborhood of $1 /|J|$ for all $i, j \in J$ for some $\epsilon>0$. Consider a new ranking problem $R^{\prime} \equiv\left\langle J^{\prime}, A^{\prime}, C^{\prime}\right\rangle$, where $J^{\prime}=J \cup\left\{j^{*}\right\}$, $A^{\prime}=a I_{J^{\prime}}$, and $C^{\prime}$ is such that $c_{i j}^{\prime} / \sum_{i \in J^{\prime}} c_{i j}^{\prime}$ is in the same $\epsilon$-neighborhood of $1 /|J|$ for every $i, j \in J$ and $\sum_{j \in J} c_{j^{*} j}^{\prime} / \sum_{i \in J^{\prime}} c_{i j}^{\prime}$ and $\sum_{i \in J} c_{i j^{*}}^{\prime} / \sum_{i \in J^{\prime}} c_{i j^{*}}^{\prime}$ are in the same $\epsilon$-neighborhood of 0 . Then, $\phi^{j^{*}}\left(R^{\prime}\right)$ must not be in the $\epsilon$-neighborhood of $\left(1 /\left|J^{\prime}\right|\right)$.

That is, moving from a system of journals with a heavy flow of mutual citations to an expanded one in which the influence of a new journal on the system and the influence of the system on the new journal are negligible, the weight assigned to the new journal should not be too large. ${ }^{3}$

Theorem 1 There is no ranking method satisfying the PV axioms and limited influence of boundary journals.

The proof is simply the combination of PV's Theorem 1 and the above example.

\section{The PV Axioms in Richer Domains}

We make two final points. The PV axioms, which look reasonable in the domain of ranking problems proposed, may not be as appealing in other domains.

(i) Suppose that one can distinguish good citations (instances in which a paper is cited like this: "we build our analysis on Smith (1776)" or "Smith (1776) is our motivation")

\footnotetext{
${ }^{1}$ The implication is that if the new journal in Example 1 is ran by a lunatic editor, who chooses to publish papers that cite only his journal (he only made one exception, publishing a paper that cited something in journal 1, only because journal 1 published one article that cites his journal), he is generously rewarded by the invariant method. Alternativley, Example 1 points to the importance of choosing the set $J$ wisely.

${ }^{2}$ This is to be distinguished from the more questionable "limited influence of journals at the frontier".

${ }^{3}$ One could think of different ways of phrasing this axiom. I chose this presentation because it is a very weak requirement. Other versions appear in Serrano and Menard (2003), and I thank Pierre Menard for his suggestion on how to appear influential. After all, if the current paper is published, Menard will have a substantial impact in economics, if measured by the invariant method.
} 
from bad citations ("Menard (1776) makes the following egregious mistake" or "For the literature to make any progress, the model in Menard (1776) must be abandoned). ${ }^{4}$

Example 2 Consider a two-journal problem, with $a_{j}=a$ for $j=1,2$ and with citation matrix having the constant $k$ in every entry. Suppose, however, that the $k$ citations made by journal 1 to journal 2 are good, while the ones made by journal 2 to journal 1 are bad. It is then clear that a method that measures "good" influence should not satisfy weak homogeneity, i.e., prescribe a relative valuation of the two journals solely on the basis of mutual citations. A similar comment applies to weak consistency, because one would have to be very careful defining the appropriate reduced problem.

(ii) There are certain contributions that are so influential that do not make it to the references section. For example, authors that work with Nash equilibrium or Arrow-Debreu economies rarely refer to the original articles. For cases like these, that one could call "textbook references" in the sense that they have already made it to the basic language of a profession, no method based on citations alone would make them justice.

\section{Concluding Remark}

I have pointed out three defficiencies of the invariant method: its poor behavior near reducible problems, its inability to distinguish between good and bad citations, and its disregard for "textbook references." Fortunately, one could think of ways to alleviate these problems, and in all cases they entail a more careful reading of the works.

\section{References}

Borges, J. L. (1962), "Pierre Menard, Author of Don Quixote," in Ficciones, English translation, Grove Press Inc., from Spanish edition, 1956.

Menard, P. (1776), An Inquiry into the Nature and Causes of the Wealth of Nations, virtual manuscript, Université Fictionale.

Palacios-Huerta, I. and O. Volij (2004), "The Measurement of Intellectual Influence," Econometrica 72, 963-978.

Serrano, R. and P. Menard (2003), "The Measurement of Intellectual Influence: the Views of Two Sceptics," mimeo.

\footnotetext{
${ }^{4}$ The above quotes are found verbatim in the literature, surprising as it may seem given that the text in Menard is absolutely identical to Smith's. The citing authors, though, rightly appreciated the important differences. See also Borges (1962).
} 
Smith, A. (1776), An Inquiry into the Nature and Causes of the Wealth of Nations, Edinburgh. 